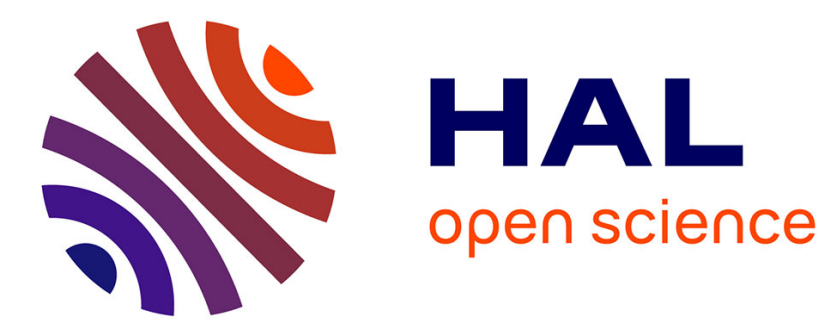

\title{
An evaluation of neurophysiological criteria used in the diagnosis of Motor Neurone Disease
}

\author{
Chris Douglass, Rosalind H Kandler, Pamela J Shaw, Christopher J \\ Mcdermott
}

\section{- To cite this version:}

Chris Douglass, Rosalind H Kandler, Pamela J Shaw, Christopher J Mcdermott. An evaluation of neurophysiological criteria used in the diagnosis of Motor Neurone Disease. Journal of Neurology, Neurosurgery and Psychiatry, 2010, 81 (6), pp.646. 10.1136/jnnp.2009.197434 . hal-00557432

\section{HAL Id: hal-00557432 \\ https://hal.science/hal-00557432}

Submitted on 19 Jan 2011

HAL is a multi-disciplinary open access archive for the deposit and dissemination of scientific research documents, whether they are published or not. The documents may come from teaching and research institutions in France or abroad, or from public or private research centers.
L'archive ouverte pluridisciplinaire HAL, est destinée au dépôt et à la diffusion de documents scientifiques de niveau recherche, publiés ou non, émanant des établissements d'enseignement et de recherche français ou étrangers, des laboratoires publics ou privés. 
An evaluation of neurophysiological criteria used in the diagnosis of Motor

Neurone Disease

Douglass $\mathrm{CP}^{1}$, Kandler $\mathrm{RH}^{2}$, Shaw $\mathbf{P J}^{3}$ and McDermott $\mathrm{CJ}^{3 *}$

${ }^{1}$ Department of Neurology, Royal Hallamshire Hospital, Sheffield

${ }^{2}$ Department of Neurophysiology, Royal Hallamshire Hospital, Sheffield

${ }^{3}$ Department of Neuroscience, Academic Neurology Unit, University of Sheffield

* Corresponding Author

Department of Neuroscience

Academic Neurology Unit

E Floor

Medical School

University of Sheffield

Beech Hill Rd

Sheffield

S10 2RX

MeSH Keywords Amyotrophic lateral sclerosis

Motor neuron disease

Neurophysiology

Electromyography

Word count 2427 


\begin{abstract}
New criteria for the neurophysiological diagnosis of Amyotrophic Lateral Sclerosis/ Motor Neurone Disease (ALS/MND) were recently proposed at an international symposium in Awaji-shima, Japan. They differ from the accepted revised El-Escorial criteria by considering fasciculation potentials to be evidence of acute denervation. In addition when assessing diagnostic certainty, the Awaji-shima criteria equate electrodiagnostic evidence of lower motor neurone dysfunction with clinical examination findings.

A retrospective review of 205 consecutive sets of notes was performed, from patients who underwent neurophysiological assessment for suspected MND. The clinical signs and neurophysiological findings were combined according to the two sets of criteria (revised El-Escorial and Awaji-shima) and the diagnoses reached were compared to the interval diagnosis, to establish the sensitivities and specificities of each protocol.

An interval diagnosis of MND was recorded in 107 patients. The sensitivity of the Awaji-shima criteria in reaching a diagnosis of MND was $60.7 \%$ and the revised El-Escorial $28 \%$, with a specificity of $95.9 \%$ for both criteria. The Awaji-shima criteria increased the sensitivity of diagnosis without affecting the specificity. Accepting EMG evidence of fasciculations as evidence of acute denervation increases the diagnostic certainty of MND and the new criteria allow earlier diagnosis of MND without increasing the false positive rate.
\end{abstract}




\section{INTRODUCTION}

Motor neurone disease (MND) is the third commonest adult onset neurodegenerative disorder, with an annual incidence of 2 per 100000 and prevalence of 5-7 per 100 000.[1] MND leads to progressive muscle weakness and atrophy, with a mixture of upper and lower motor neurone signs, due to degeneration of pyramidal neurons in the motor cortex, cranial motor neurons and anterior horn cells in the spinal cord. Classical MND tends to be focal in onset, with a particular group of motor neurones affected first. This can lead to a delay in diagnosis, as early in the disease course there may be few clinical signs. An earlier diagnosis reduces the period of diagnostic uncertainty for the patient and allows the timely planning of future care and support. Additionally commencing neuroprotective agents, such as Riluzole, whilst there is a larger pool of surviving motor neurones may improve any therapeutic effect.[1] Neurophysiological evaluation is used in patients with suspected MND to both support the diagnosis, by finding evidence of a more widespread motor neurone dysfunction than is apparent clinically, and also to exclude other disorders which may mimic MND. Several sets of criteria have been proposed over the recent past, which combine the clinical evaluation and neurophysiological assessment to facilitate an earlier diagnosis of MND.

Lambert suggested that clinical neurophysiology could assist in the diagnosis of ALS and in the exclusion of other peripheral neuromuscular pathologies in two papers in 1956 and 1957 and later published a list of criteria for the electrophysiological confirmation of ALS in 1969.[2] In 1990 a three day workshop on "The clinical limits of ALS" was convened at El Escorial, Spain by the World Federation of Neurology Subcommittee on Motor Neuron Disease. Their aim was to develop diagnostic criteria which would include electrophysiological and clinical data, and be workable, internationally acceptable and provide an algorithm which would enhance clinical studies, therapeutic trials and molecular genetic research 
studies.[4] These criteria were revised in 1998 at a workshop in Airlie House, Warrenton, Virginia.[5]

The revised El Escorial/Airlie House criteria are summarised in Figure 1a. The body is divided into 4 regions, the brainstem, and the cervical, thoracic and lumbosacral spinal cord regions. The revised El Escorial criteria categorise the patients into 4 levels of diagnostic probability; Clinically definite ALS, Clinically probable, Clinically probable- laboratory supported (a category not present in the original El Escorial criteria), and Clinically possible ALS. The revised El Escorial criteria have been criticised for being too strict, resulting in some patients with clinically obvious MND never achieving a diagnostic category higher than Clinically Possible. One area for debate regarding the revised El-Escorial criteria has been the fact that fasciculations on electromyography (EMG) are not taken as evidence of lower motor neurone dysfunction. Lambert stated that "The EMG discloses fasciculations so regularly in ALS that one rarely accepts the diagnosis unless fasciculation is present".[2] The revised El Escorial criteria stated that fasciculation potentials were a characteristic clinical feature of ALS and that their absence raises diagnostic doubts, however they are not accepted as evidence of active denervation. Wilbourn suggested that the El Escorial criteria should be revised within a less rigid framework so that fasciculations can be given the emphasis they merit.[3]

In 2006 an international symposium was held in Awaji-shima, Japan to determine the best use and interpretation of electrophysiological data in the diagnosis of ALS.[6] The Awaji-shima criteria proposed that electrophysiological and clinical signs of lower motor neurone (LMN) degeneration are given equal weight in the decision as to which diagnostic category the patient is assigned. The revised El Escorial criteria do not allow the EMG and clinical abnormalities to be combined in a single limb, a limb must be determined to be abnormal by one technique or the other. The Awaji-shima criteria, by accepting neurogenic EMG abnormality as equivalent to clinical abnormality, allows a currently strong limb to be classified as abnormal 
earlier than if the decision were based on EMG or clinical criteria alone.[6] This renders the clinically probable-laboratory supported ALS category redundant as all categories can now use laboratory support in the diagnosis.

In both sets of criteria EMG evidence of lower motor neurone involvement requires signs of both active and chronic denervation to be present. The presence of chronic denervation is confirmed by long duration, large amplitude, polyphasic, unstable motor unit potentials and decreased motor unit recruitment. Active denervation is confirmed by the presence of positive sharp waves and fibrillation potentials. These changes must be present in at least two muscles in the cervical and lumbosacral spinal cord regions and in one muscle in the brainstem and thoracic cord regions for the regions to be categorised as affected. The criterion for numbers of muscles involved in a region is shared by both the El Escorial and Awaji-shima groups and has been shown to achieve the best combination of sensitivity and specificity.[7]

A further significant difference in the Awaji-shima criteria, is the reintroduction of fasciculation potentials as evidence of acute denervation, equivalent to that of fibrillation potentials and positive sharp waves. This is similar to the Lambert criteria and it has been suggested that these changes will increase the sensitivity of the diagnostic criteria for ALS without changing the specificity.[8] The El-Escorial criteria did not include fasciculations as part of the diagnostic criteria as they were considered to be less specific than fibrillations and could produce false positive results. The fasciculations that are accepted in the Awaji-shima criteria are not the simple and stable fasciculations that may occur in benign conditions but unstable, complex fasciculation potentials in the presence of unstable motor unit potentials in the context of suspected ALS. 


\section{AIMS}

The aim of this study was to determine the sensitivity and specificity of the Awajishima criteria for diagnosing MND in a clinical/ non research setting as compared to the revised El Escorial criteria.

\section{METHODS}

The neurophysiology department database was interrogated to identify 250 consecutive patients, who had been referred from January $1^{\text {st }} 2000$ with motor neurone disease included in the differential diagnosis. This time period was chosen as it would allow sufficient time for the interval diagnosis to become clinically evident. 205 patients had full clinical records and EMG results available. The results of the electromyography and nerve conduction studies were re-examined and changes of chronic denervation and of active denervation (fibrillation potentials and positive sharp waves and/or complex fasciculations) in each area of the body were recorded. The nerve conduction studies were recorded as normal/ supportive of MND or the alternative diagnosis was recorded. All patients included in the study had a proximal and a distal muscle in an upper and lower limb sampled and a bulbar muscle was also examined where indicated. A sensory and motor nerve conduction study was performed in an upper and lower limb.

The clinical notes were examined. The clinical signs on presentation were recorded as positive or negative for upper motor neurone and/or lower motor neurone signs in each area of the body. The clinical diagnosis from the last review was recorded as the interval diagnosis.

The clinical findings and neurophysiology results were then combined according to the two sets of criteria, and the diagnosis that would have been reached after the first clinical encounter and electro-diagnostic test, was recorded as the criteria diagnosis. The criteria diagnoses were then compared to the interval diagnoses and the sensitivity and specificity of the Awaji-shima and revised El-Escorial criteria 
were calculated. For the purpose of calculating the sensitivities and specificities, if the criteria diagnosis was clinically definite MND or clinically probable/ clinically probable-laboratory supported MND then these patients were grouped together as having a "positive MND criteria diagnosis". Conversely if the criteria diagnosis was clinically possible MND or the patient did not achieve a diagnostic category then these patient were assigned a "negative MND criteria diagnosis".

\section{RESULTS}

The clinical notes and the corresponding neurophysiology reports of 205 patients referred for EMG with suspected MND were reviewed. The interval diagnosis was MND in 107; of these 97 were limb onset amyotrophic lateral sclerosis and 10 were bulbar onset ALS. 3 patients had a family history of MND but were all SOD1 negative cases. The interval diagnosis in the remaining cases was varied. The largest groupings were neuropathies (22), radiculopathies (19), and benign cramp fasciculation syndrome (6). The remaining 51 had no evidence of neurological disease or a wide variety of disorders, with no suggestion of MND. The mean duration between initial clinical presentation and assessment, and assigning the interval diagnosis in this study was 89 months (range 65 to 116 months). The mean age of patients with MND was 64.3 (range 31 to 95). The mean duration of symptoms at the time of initial clinical presentation was 17.9 months (range 1 to 192 months) and mean time from clinical presentation to EMG was 1.2 months (range 0 to 37 months). Mean length of follow up was 16.3 months (range 1 to 87 months). There was an approximate 3:1 male to female ratio.

On clinical examination alone prior to the neurophysiological assessment, using the clinical El Escorial criteria, 21 patients had a clinically probable and 12 patients had a clinically definite diagnosis of ALS. These 33 patients were classed as having a positive MND criteria diagnosis. 102 were assigned to the clinically possible ALS category and 70 patients could not be assigned a diagnostic category. These 172 
patients were grouped together as considered to have a negative MND criteria diagnosis.

When the EMG information, interpreted as per the revised El Escorial criteria, was added to the clinical information, there were 22 patients with clinically probable and 12 with clinically definite MND, giving a total of 34 patients with a positive MND criteria diagnosis. 101 patients had clinically possible MND and there were 70 patients who did not reach a diagnostic category, making a total of 171 patients in the negative MND criteria diagnosis group.

Using the Awaji-shima criteria the number of patients assigned a positive MND criteria diagnosis rose to 69 (clinically definite 15, clinically probable 54) and the number in the negative MNG criteria diagnosis group fell to 136 (clinically possible 67 , those outside of a diagnostic category 69). The number of patients attaining a positive criteria diagnosis which may allow entry into a clinical trial or the commencement of therapy is approximately double that of the El Escorial criteria. See figure 2 .

Using the numbers of patients with a positive or negative MND criteria diagnosis and the numbers of those patients who had a final interval clinical diagnosis of ALS or another diagnosis (table 1), we were able to calculate the sensitivity and specificity of the two sets of criteria. Using the clinical information of the upper and lower motor signs found on examination, interpreted using the revised El Escorial clinical criteria, to make the diagnosis of MND had a sensitivity of $27.1 \%$. When information from the EMG was applied using the revised El Escorial criteria, the sensitivity rose to $28 \%$. Using the Awaji-shima criteria however increased the sensitivity to $60.7 \%$. The specificity of each of the three methods was $95.9 \%$ indicating that there were no more false positives with the new criteria. 


\section{DISCUSSION}

The Awaji-shima criteria have been shown to increase the sensitivity of diagnosis of MND compared to the revised El Escorial criteria, without an increase in the false positive rate. There were 4 patients who had a diagnosis of clinically probable ALS despite their final clinical diagnosis not being ALS. They gained this false positive diagnosis through the clinical signs (upper and lower motor neurone signs in 2 regions of the body due to degenerative spinal cord disease) rather than through the extra information gained from the neurophysiology testing.

The Awaji-shima criteria would have categorised 35 more patients as having a positive diagnosis than the El Escorial criteria (69 vs 34 had a diagnosis of clinically definite or probable). These 35 patients could have been entered into clinical trials at this point and neurologists investigating these patients may have felt more confident in giving a definite diagnosis to the patients and initiating Riluzole and supportive interventions. In January 2009 De Cavalho and Swash suggested that the Awaji-shima algorithm superimposed onto the El Escorial criteria in a research setting created an increased sensitivity of diagnosis.[9] The sensitivity was increased to $95 \%$ using the Awaji-shima criteria compared to 53\% when applying the revised El Escorial criteria. They studied 55 consecutive patients previously diagnosed with ALS by standard clinical techniques in whom neuropathies had been excluded by sensory and motor nerve conduction studies. They performed extensive EMG studies in at least 10 sites in each limb and cranially innervated muscle. Additionally, in 44 of the 55 patients the diaphragm and a trunk muscle were also studied. This level of investigation in a selected group of patients in a research setting may account for the higher sensitivity than we achieved in our less selected clinical group of patients. The specificity of the criteria was not investigated in their paper however and so our study is the first to demonstrate that the criteria are specific even in a clinical setting with standard neurophysiological testing. Using the Awaji-shima criteria facilitates an earlier 
diagnosis in patients with MND. This may have benefits in reducing periods of diagnostic uncertainty, reduce repetitive testing, enable earlier recruitment into clinical trials and commencement of supportive treatments and Riluzole. 


\section{Acknowledgements}

The authors would like to thank Drs John Jarratt and Arup Chattopadhyay for the neurophysiological testing of patients, the Sheffield neurologists for allowing access to the notes of their patients and their secretaries for assistance in obtaining the notes.

\section{Competing interests.}

None.

\section{Funding.}

None.

The Corresponding Author has the right to grant on behalf of all authors and does grant on behalf of all authors, an exclusive licence (or non-exclusive for government employees) on a worldwide basis to the BMJ Publishing Group Ltd, and its Licensees to permit this article (if accepted) to be published in JNNP and any other BMJPGL products and to exploit all subsidiary rights, as set out in our licence. 


\section{REFERENCES}

[1] McDermott CJ and Shaw PJ. Diagnosis and management of motor neurone disease. BMJ 2008;336:658-662

[2] Lambert EH. Electromyography in amyotrophic Lateral Sclerosis. In : Norris FH, Kurland LT, editors. Motor Neuron Diseases. New York: Grune and Stratton, 1969:135-53.

[3] Wilbourn AJ. Clinical neurophysiology in the diagnosis of amyotrophic lateral sclerosis: The Lambert and the El Escorial criteria. J Neurol Sci 1998 (Suppl):25-29. [4] Brooks BR. El Escorial World Federation of Neurology criteria for the diagnosis of amyotrophic lateral sclerosis. Subcommittee on Motor Neuron Diseases/Amyotrophic Lateral Sclerosis of the World Federation of Neurology Research Group on Neuromuscular Diseases and the El Escorial Clinical limits of amyotrophic lateral sclerosis workshop contributors. J Neurol. Sci 1994;124 (Suppl):96-107

[5] Brooks BR, Miller RG, Swash M, Munsat TL. El Escorial revisited: revised criteria for the diagnosis of amyotrophic lateral sclerosis. Amyotroph Lateral Sclerosis Other Motor Neuron Disorders. 2000;1:293-9

[6] de Carvalho M, Dangler R, Eisen A, England JD, Kaji R, Kimura J, Mills K, Mitsumoto H, Nodera H, Shefner J, and Swash M.

Electrodiagnostic criteria for diagnosis of ALS. Consensus of an international Symposium sponsored by IFCN. December 3-5 2006, Awaji-shima, Japan.

[7] Makki AA and Benatar M. The electromyographic diagnosis of amyotrophic lateral sclerosis: does the evidence support the El Escorial criteria? Muscle \& Nerve 2007; 35: 614-619.

[8] Fuglsang-Friedericksen A. Editorial. Diagnostic criteria for amyotrophic lateral sclerosis (ALS). Clinical Neurophysiology 2008; 119: 495-496. 
[9] DeCarvalho M and Swash M. Awaji diagnostic algorithm increases sensitivity of El Escorial criteria for ALS diagnosis. Amyotrophic Lateral Sclerosis; 2009; 10:53-

57. 


\section{LEGEND FOR FIGURES}

\section{Figure 1a}

Revised El Escorial criteria

Figure $1 b$.

Awaji shima critera

\section{Figure 2}

Diagnostic categories of patients with confirmed ALS as determined by the criteria.

\section{TABLES}

\section{Table 1}

Numbers of patients assigned to each diagnostic category

\section{Table 2}

Sensitivity and specificity of the diagnostic criteria. 
Table 1. Numbers of patients assigned to each diagnostic category

\begin{tabular}{|c|c|c|c|c|c|}
\hline \multirow[b]{2}{*}{ Awaji Shima } & \multirow[b]{2}{*}{ Def } & \multicolumn{2}{|c|}{ ALS + } & \multicolumn{2}{|c|}{ ALS- } \\
\hline & & 15 & 65 & 0 & 4 \\
\hline & Prob & 50 & & 4 & \\
\hline & Poss & 29 & 42 & 38 & 94 \\
\hline & Other & 13 & & 56 & \\
\hline \multirow[t]{4}{*}{ El Escorial } & Def & 12 & \multirow[t]{2}{*}{30} & 0 & \multirow[t]{2}{*}{4} \\
\hline & Prob & 18 & & 4 & \\
\hline & Poss & 64 & 77 & 37 & 94 \\
\hline & Other & 13 & & 57 & \\
\hline \multirow{4}{*}{$\begin{array}{l}\text { Pretest clinical } \\
\text { data only }\end{array}$} & Def & 12 & \multirow[t]{2}{*}{29} & 0 & \multirow[t]{2}{*}{4} \\
\hline & Prob & 17 & & 4 & \\
\hline & Poss & 66 & \multirow[t]{2}{*}{78} & 36 & \multirow[t]{2}{*}{94} \\
\hline & Other & 12 & & 58 & \\
\hline
\end{tabular}


Table 2. Sensitivity and specificity of the diagnostic criteria.

\begin{tabular}{|l|c|c|}
\hline & Sensitivity & Specificity \\
\hline Pre-EMG diagnosis & $27.1 \%$ & $95.9 \%$ \\
\hline El Escorial criteria & $28.0 \%$ & $95.9 \%$ \\
\hline Awaji Shima criteria & $60.7 \%$ & $95.9 \%$ \\
\hline
\end{tabular}




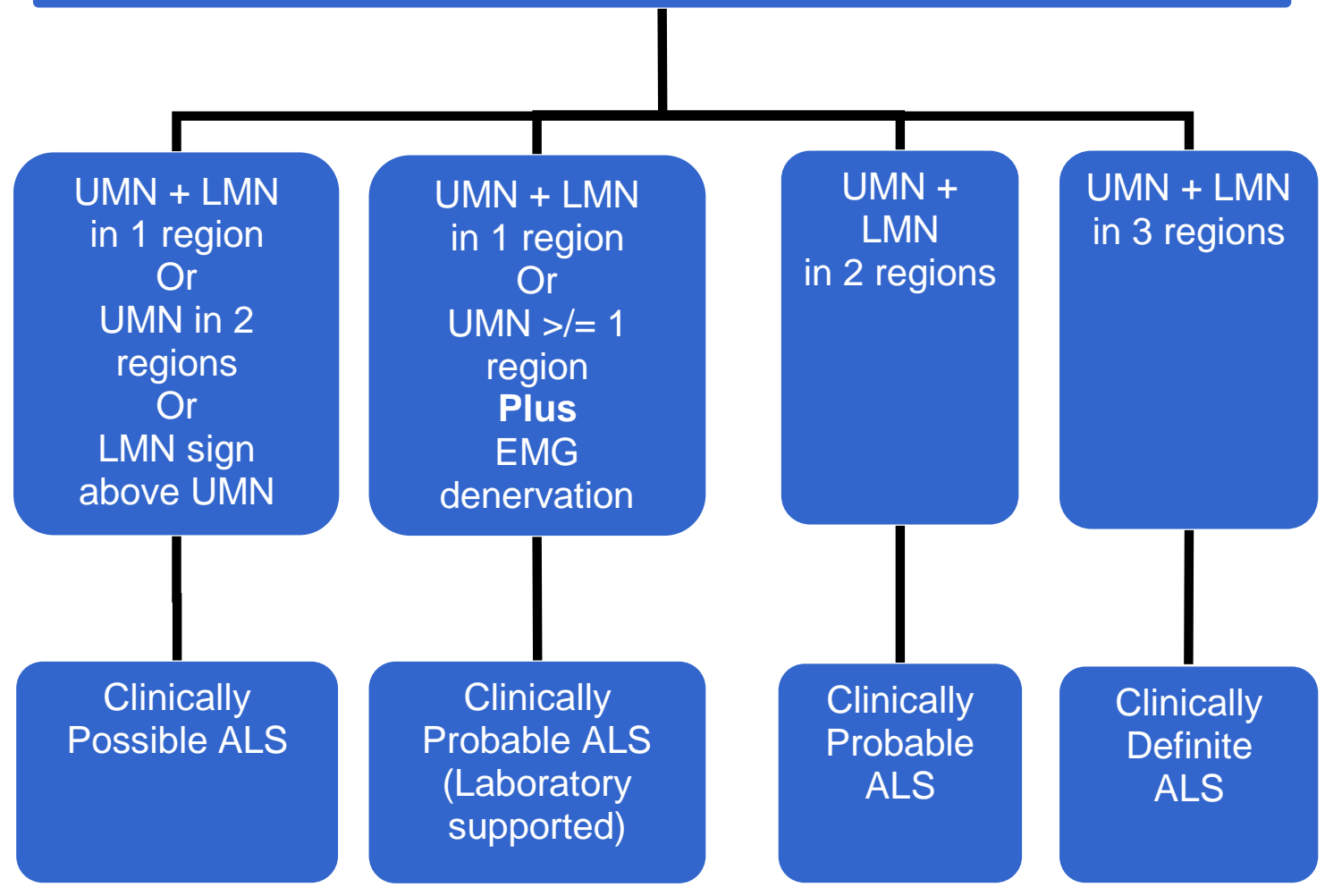




\section{Awail- Shima criteria}

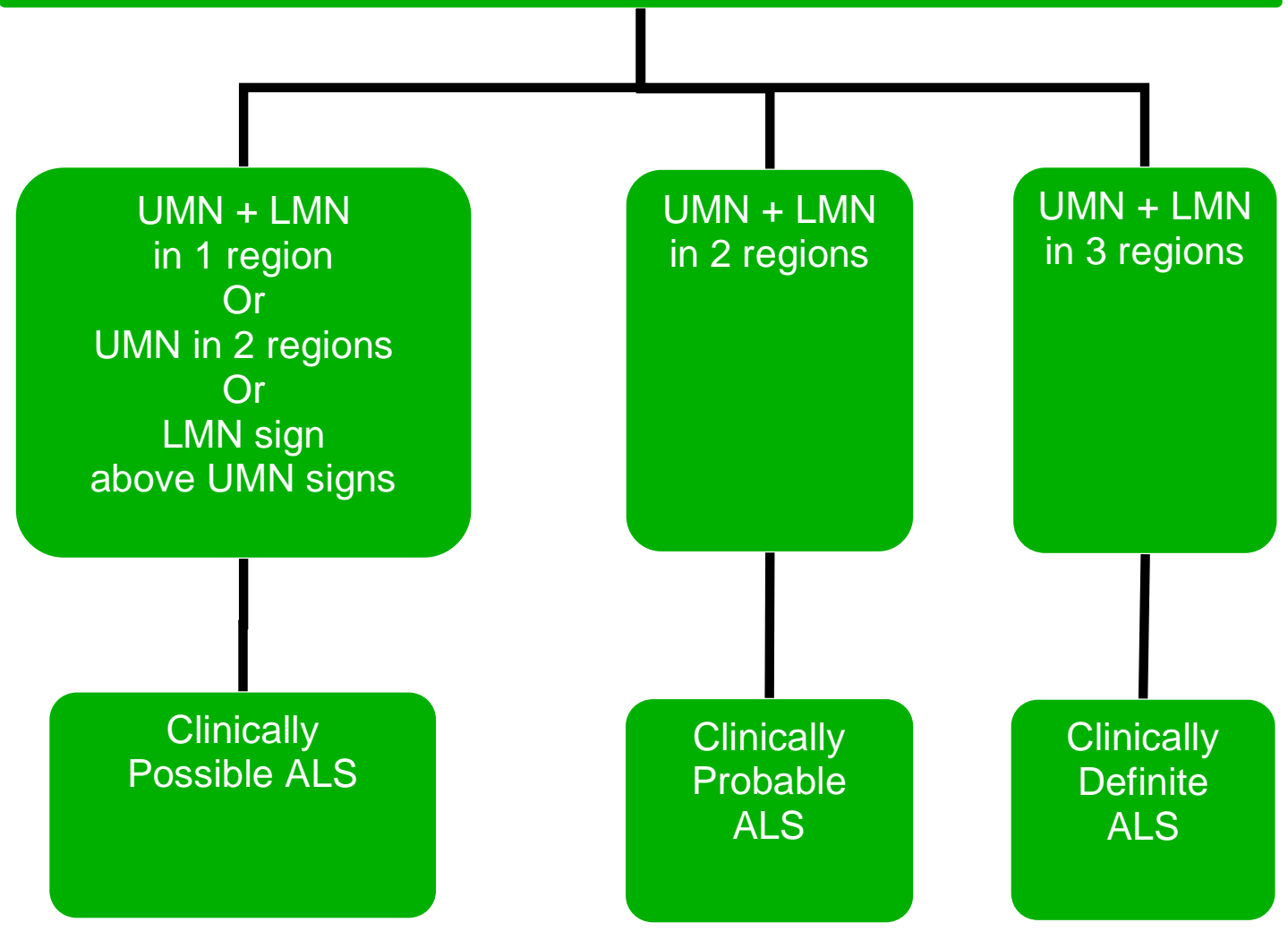




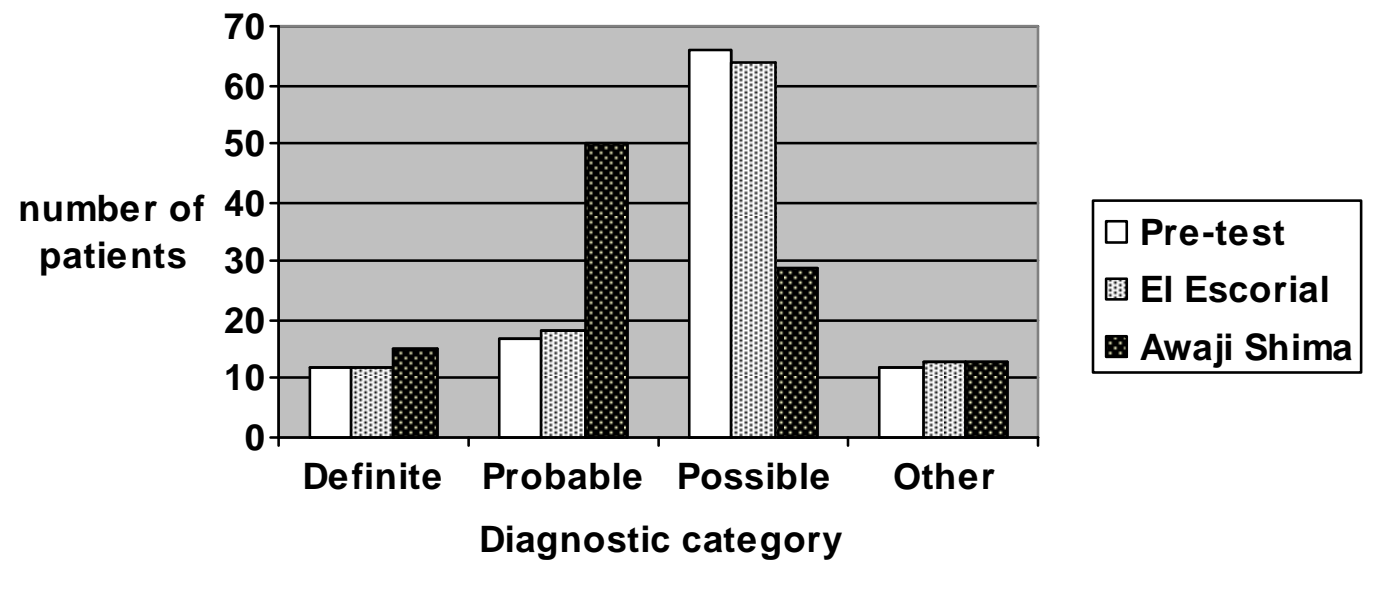

